PSFC/JA-06-13

\title{
A Comparison of Measured and Calculated Toroidal Alfvén Eigenmode Damping Rates in Alcator C-Mod
}

\author{
J A Snipes, N N Gorelenkov*, J A Sears \\ MIT Plasma Science and Fusion Center, Cambridge, MA 02139 \\ USA \\ *Princeton Plasma Physics Laboratory, Princeton, NJ 08543 USA
}

\author{
Plasma Science and Fusion Center \\ Massachusetts Institute of Technology \\ Cambridge MA 02139 USA
}

This work was supported by the U.S. Department of Energy, Grant No. DE-FC0299ER54512. Reproduction, translation, publication, use and disposal, in whole or in part, by or for the United States government is permitted. 


\title{
A Comparison of Measured and Calculated Toroidal Alfvén Eigenmode Damping Rates in Alcator C-Mod
}

\author{
J A Snipes, N N Gorelenkov*, J A Sears \\ MIT Plasma Science and Fusion Center, Cambridge, MA 02139 USA \\ *Princeton Plasma Physics Laboratory, Princeton, NJ 08543 USA
}

\begin{abstract}
Measured damping rates of stable Toroidal Alfvén Eigenmodes (TAEs) have been compared with damping rates calculated with the NOVA-K code for a number of experimental conditions on Alcator C-Mod in an attempt to validate the NOVA-K damping model. Very low amplitude ( $\tilde{B}_{\theta} / B_{\theta} \sim 10^{-7}$ at the wall) stable TAEs are excited in Ohmic plasmas with a pair of active MHD antennas inside C-Mod. By sweeping the excited frequency of the antennas through the expected TAE frequency, the frequency width of a mode resonance can be measured with poloidal field pick-up coils on outboard limiters to determine the damping rate of that mode. The calculated equilibrium and main plasma parameters at the time of the resonance are input into the NOVA-K code to then calculate the expected damping rate for the measured toroidal mode number and compare with the experimental value. The calculations include continuum damping, Landau damping on both the electrons and the background and impurity ions, and collisional damping on the electrons. Comparisons have been made for inner wall limited and lower single null diverted cases over a range of ITER-relevant moderate toroidal mode numbers $(3 \leq|n| \leq 9)$. Good agreement between the experiment and theoretical calculations can be obtained with reasonable q profiles for the plasma conditions in which perturbative NOVA models are applicable, but the results are found to depend very sensitively on the assumed $q$ profile.
\end{abstract}

\section{Introduction}

Fast particle driven unstable modes such as Toroidal Alfvén Eigenmodes (TAEs) [1-5] are found in most magnetic confinement devices in the presence of energetic particles driven by auxiliary heating and under some conditions eject the fast particles, reducing the plasma performance or even causing damage to the first wall. Calculations predict that moderate toroidal mode number $(n \sim 10)$ TAEs will be unstable in ITER $[6,7]$ and, if they grow large enough, could quench the fusion burn or cause damage to the first wall. Understanding the physics of these modes is important to learn how to avoid or control them to better control the fusion burn in next step devices. TAEs become unstable when their energetic particle drive exceeds the combination of 
multiple damping mechanisms [8-10]. While the energetic particle drive is believed to be well understood [11-13], the damping mechanisms are complex and the theory needs to be quantitatively benchmarked with experiment, particularly in the range of medium to high toroidal mode numbers expected in ITER.

Previous experiments carried out on JET with very large low $n \leq 2$ antennas have excited stable TAEs in Ohmic plasmas over a wide range of conditions [14-16]. Comparisons of the measured damping rate were made with NOVA-K calculations with the result that NOVA-K under predicted the measured damping rate by more than an order of magnitude using measured density and safety factor profiles [17]. It is noteworthy that special studies have been performed recently to understand those results. Two different groups came to the same conclusion that the dominant contribution to the damping of $n=1$ TAEs in JET can come only from the plasma edge $[18,19]$ and is mostly due to the continuum damping, whereas radiative damping was shown to be small.

To better understand the physics of Alfvén eigenmodes, with particular emphasis on the range of modes expected to be unstable in ITER, a pair of active MHD antennas was installed in Alcator C-Mod to excite a broad range of moderate $|n|<16$ stable AEs [20]. A number of experiments have been performed exciting AEs and measuring their damping rates in C-Mod Ohmic plasmas with inner wall limited and lower single null magnetic configurations [21,22]. While the measured damping rates $(0.1 \%<\gamma / \omega<4 \%)$ for these moderate $n$ TAEs were in the same range as for the low $n$ TAEs in JET, some differences in the scaling of the damping rate with plasma shaping and magnetic configuration were found. In particular, the lowest damping 
rates for moderate $n$ TAEs in C-Mod were found in lower single null diverted plasmas with small outer gaps, while diverted plasmas had the largest damping rates for low $n$ AEs in JET. Given these measured differences and the fact that moderate $n$ AEs will couple a larger number of poloidal harmonics with different radial distributions, the physics of the damping of moderate $n$ AEs may be significantly different from that of low $n$ AEs.

To investigate the physics of moderate $n$ AEs, the NOVA-K code [23] has been used to calculate moderate $n$ damping rates and compare with the damping rates of stable TAEs measured in C-Mod. Improvements have been made in the damping rate calculations in NOVA$\mathrm{K}$ [24], particularly with regard to continuum damping [25], which usually turns out to be the dominant damping mechanism for the cases that have been considered. Nevertheless the measured damping rate in absolute value is often low enough, 1-3\%, to justify the applicability of the perturbative model used in NOVA-K. The other damping mechanisms that are included in these studies are collisional damping on the electrons and Landau damping on the thermal electrons, deuterium ions, and carbon ions as the only assumed impurity. Radiative damping has not been included. The purpose of this paper is to compare for the first time moderate- $n$ TAE measured and simulated damping rates under conditions in which the NOVA-K model is applicable.

The next section of this paper describes the active MHD antennas on C-Mod and accompanying magnetic pick-up coils used for exciting and measuring the AE resonances, respectively. Section 3 gives a brief description of the NOVA-K code. Section 3.1 gives the results of the comparison of the calculated and measured damping rate for an $n=4$ inner wall 
limited case. Section 3.2 compares the calculated and measured damping rates for an $n=-4$

lower single null case. Section 3.3 concentrates on an $n=9$ lower single null case with artificially varied safety factor profile to systematically study the effect of small changes in q on the calculated damping rate.

\section{Active MHD Experiments on C-Mod}

The active MHD system on C-Mod for measuring the damping rates of stable Alfvén eigenmodes consists of a pair of antennas above and below the outboard midplane at one toroidal location and 65 poloidal field pick-up coils mounted to outboard limiters at two toroidal locations. Figure 1a shows a cross-section of the plasma and a drawing of the active MHD antennas. For a typical plasma shape, there is only a $2.5 \mathrm{~cm}$ outer gap between the antennas and the last closed flux surface. Figure $1 \mathrm{~b}$ also shows a photo of the antennas and one of the outboard limiters, where pick-up coils are mounted to the sides and beneath the tiles. Figure 1c also shows a geometric toroidal mode number spectrum of each of the antennas, indicating a broad spectrum with a full-width-half-maximum of $|n| \approx 16$. With only one set of antennas toroidally, no selection of toroidal mode numbers can be made and there can be no directionality of the excited waves.

Two amplifiers [26] can drive the antennas independently with up to $25 \mathrm{~A}$ of current across the AE frequency range from $100 \mathrm{kHz}-1 \mathrm{MHz}$, which produces a radial field perturbation of about $10^{-4} \mathrm{~T}$ at the typical $\mathrm{q}=1.5$ surface. Note that while the total perturbed field is of order $10^{-4} \mathrm{~T}$, the stable TAE resonances measured with the poloidal field pick up coils at the

wall are typically $\tilde{B}_{\theta}<10^{-6} \mathrm{~T}$, so that only the most sensitive fluctuation diagnostics can observe 
these modes. Such stable TAE resonances have not been observed on the line integrated density fluctuation measurements along vertical chords through the plasma core with Phase Contrast Imaging (PCI) [27].

In early experiments, current was driven in only the upper antenna while in more recent experiments, both antennas were driven in phase. Through synchronous detection of the pick-up coil signals with the antenna current, a peak in the amplitude combined with a circular sweep of the phase of the real and imaginary parts of the transfer function indicate clear resonances as the frequency sweeps through the expected frequency of the center of the TAE gap given approximately by $\omega_{T A E}=\mathrm{v}_{A} / 2 q R$ where $\mathrm{v}_{A}=B / \sqrt{4 \pi m_{i} n_{i}}$ is the Alfvén velocity. Resonances were observed to fit this simple expression evaluated using the major radius of the magnetic axis, the toroidal field on axis, the core line averaged electron density in place of the ion density, 2.1 for the effective ion mass, and the q value taken at 1.5. Early experiments relied on ramping the toroidal field at constant antenna frequency to determine the width of the resonance by effectively sweeping the plasma TAE frequency through the antenna frequency. To improve the time resolution of the damping rate measurements, the frequency of the antennas can be swept back and forth through the above TAE frequency calculated in real time with analog line averaged electron density and toroidal field signals and assumed values for the other parameters. This allows resonances to be measured typically every $100 \mathrm{~ms}$ during the discharge. The typical uncertainty in the measured damping rate is $\pm 20 \%$.

While the measured resonance frequency is very precise, the actual mode frequency in the plasma frame requires knowledge of the plasma rotation profile. There is a three radial 
position $\mathrm{x}$ ray crystal spectrometer on C-Mod [28] that measures the toroidal rotation of argon impurities in the plasma. For standard Ohmic plasma conditions as in these experiments, the plasma toroidal rotation profile is usually rather flat in the range of $2 \mathrm{kHz}$ to $5 \mathrm{kHz}$ in the electron drift direction, opposite to the plasma current. For a TAE rotating in the ion drift direction, the plasma rotation will then reduce the measured frequency of the mode by $n$ times the plasma toroidal rotation frequency. So, the mode frequency in the plasma frame will be higher than the measured frequency by $n \omega_{\varphi}$. In some cases, the $\mathrm{x}$ ray crystal spectrometer data were unavailable, so the plasma rotation can only be estimated and for moderately high $n$ numbers, this can lead to a significant uncertainty in the mode frequency in the plasma frame.

Three closely spaced $\left(\Delta \varphi \approx 2.5^{\circ}\right)$ pick-up coils $10 \mathrm{~cm}$ above and below the midplane on each of the two outboard limiters [18] provide different toroidal phases of the modes to determine their toroidal mode numbers. These are particularly well placed for measuring the moderate $n$ numbers excited by the active MHD antennas. By fitting the phases of the residues of the transfer functions of the toroidally displaced coils, the toroidal mode number of a given resonance can be determined. Although the antennas drive a broad spectrum of modes, the observed plasma resonances usually consist of a single toroidal mode number that typically falls in the range of $3 \leq|n| \leq 14$.

\section{NOVA-K TAE Damping Rate Calculations}

The NOVA-K code was used to calculate the damping rate for TAEs for a range of conditions in C-Mod Ohmic plasmas. The damping terms in these calculations included continuum damping, collisions with electrons [24], and Landau damping on the thermal electrons, the deuterium ions, 
and on the carbon ions [23], which is the only assumed impurity. Note that boron is the dominant impurity in C-Mod, but this is not expected to significantly affect the results of this paper. Recently added damping on the Alfvén continuum [25] is based on the perturbative technique [29] in which the mode is assumed to be radially localized in the TAE gap and interact with the continuum only at the tail (evanescent region) of its radial structure. In such case, the damping is expected to be small and its absolute value is a criterion for the applicability of the model. The NOVA-K continuum damping model has not been extensively compared with experiments yet and was applied only in connection with the study of the AE propagation through the continuum [25]. Thus, this comparison is important to help validate NOVA-K models. While there is a model for radiative damping in the code [24], it is only valid for circular geometry and low magnetic shear conditions, which is not the case in these experiments, so radiative damping was not included. Measured values of $\mathrm{Z}_{\text {eff }}$ were used to determine the impurity concentration. The fast particle effects available in NOVA-K were artificially turned off in the code to compare with these Ohmic conditions where no significant fast ion tail is present. The code was run in free-boundary mode, which allows the eigenfunction of the perturbation to have finite amplitude at the plasma boundary, since in the experiment, the mode is measured with pick-up coils mounted to the outboard limiters well outside the last closed flux surface. CMod temperature and density profiles from Thomson scattering and EFIT [30] calculated equilibrium parameters were input into TRANSP [31]. The output of TRANSP, including the calculated total plasma $\beta$, was then used as input into NOVA-K at a given point in time in the discharge when a stable TAE resonance was observed. The measured $n$ number from the phase difference of several toroidally displaced pick-up coils was then used in NOVA-K to search for modes near the measured resonant frequency, taking into account the Doppler shift, $n \omega_{\varphi}$, of the 
core plasma rotation. For the measured moderate $n$ numbers, as expected from theory, there are a number of eigenmode solutions in the TAE gap at several frequencies in the range of the measured frequency. Their radial eigenfunctions are then analyzed to determine whether or not there is strong interaction with the continuum. For the modes that do not exhibit strong interaction with the continuum $\left((\gamma / \omega)_{\text {continuum }}<5 \%\right)$, the additional damping terms were calculated and summed to compare the total damping rate with the experimentally measured one.

Several Ohmic plasma conditions were modeled including inner wall limited and lower single null diverted plasmas. All of these plasmas were sawtoothing indicating that the central $\mathrm{q}$ value $\sim 1$. Plasmas with measured damping rates from $0.76 \%<|\gamma / \omega|<3.0 \%$ and n numbers between $3 \leq|n| \leq 9$ were chosen for this comparison. At present, the q profile is not measured but has been calculated with EFIT and TRANSP to give an estimate based only on external magnetic measurements. The sensitivity to this calculated q profile was examined by running NOVA-K three times with 0.9, 1.0, and 1.1 multiplying the EFIT q profile for one case.

\subsection{Inner wall limited plasma with $n=4$ stable TAE and ramping toroidal field}

Figure 2 shows the plasma cross-section for an inner wall limited plasma and the spectrogram of a magnetic pick-up coil signal as the toroidal field is ramped so that the center of the TAE gap frequency for $\mathrm{q}=1.5\left(\omega_{T A E}(q=1.5)=\mathrm{v}_{A} / 3 R\right)$ passes through the constant active MHD antenna frequency, which was set to $420 \mathrm{kHz}$. The plasma current and electron density were nearly constant at $\mathrm{I}_{\mathrm{p}}=0.82 \mathrm{MA}$ and $\bar{n}_{e}=3 \times 10^{20} \mathrm{~m}^{-3}$. The toroidal field ramped from $6.6 \mathrm{~T}$ down to 5.4 $\mathrm{T}$ during this time window so that $\mathrm{q}_{95}$ ramped from 4.5 down to 3.7. A clear peak is visible 
in the magnetic pick-up coil signal shortly after the TAE frequency passes through the active MHD frequency. The real and imaginary parts of the transfer function of the pick-up coil signal to the antenna current map out a circle in the complex plane. The fitted damping rate came out to $\gamma / \omega \sim-3 \%$. The phases of the residues of the transfer functions of three closely toroidally spaced pick-up coil signals gives a linear fit to $n=4$ in this case. Note that this is in the countercurrent direction since the plasma current direction is negative (clockwise) when viewed from above.

The NOVA-K code was run for this case with two different q profiles beginning with the EFIT calculated q profile based on magnetic measurements compared with an artificially calculated flat q radial profile out to $\sqrt{\Psi_{p o l}} \sim 0.7$, where $\Psi_{p o l}$ is the normalized poloidal flux (Figure 3). The plasma rotation was not measured for this case, but under similar conditions, the core ion rotation is typically $<5 \mathrm{kHz}$ in the electron direction (counter-current) so the mode frequency in the plasma frame for $n=4$ taking into account the Doppler shift would be $\mathrm{f}_{\text {plasma }}=$ $\mathrm{f}_{\text {meas }}+n \mathrm{v}_{\varphi} / 2 \pi \mathrm{R}=420 \mathrm{kHz}+20 \mathrm{kHz}$. Modes near this frequency with $n=4$ were computed for both q profiles without strong continuum interactions. Table 1 shows the calculated damping rates for the modes that were found near this frequency for both q profiles with the contributions from electron collisions, Landau damping on the thermal electrons, deuterium and carbon ions, and continuum damping indicated separately. The mode without strong continuum interaction that is closest to the experimentally measured frequency is with the EFIT q profile and has a frequency of $427.4 \mathrm{kHz}$ and a total damping rate of $-0.46 \%$. This is considerably lower in absolute value than the measured damping rate of $-3 \%$. Two modes without strong continuum interaction were found with the flat q profile at $457.2 \mathrm{kHz}$ and $476.7 \mathrm{kHz}$ with calculated 
damping rates of $-0.19 \%$ and $-1.62 \%$, respectively. The latter damping rate is within a factor of two of the measured damping rate.

The radial structures of the poloidal harmonics of the TAE eigenfunction for the 427.4 $\mathrm{kHz} n=4$ mode with the EFIT q profile are shown in Figure 4a. This is the normal to the magnetic surface component of the plasma displacement vector for each poloidal harmonic. The profile of the Alfvén eigenmode gap structure is also shown in Figure 4b. The gap is nearly closed in the core and there is strong continuum interaction from about the mid radius inward, but the dominant part of the mode is outside of the mid radius where the continuum interaction is weak. So, the total damping rate is not very large with $\gamma / \omega=-0.46 \%$.

For TAE modes, the dominant contribution to the continuum damping is typically coming from the edge of the plasma, even though the interaction with the continuum in the plasma center based on the mode structure seems to be strong. This is because such damping gets small if the magnetic shear is low [29], such as in the center. For the mode presented in Figure 4a, the interaction is much stronger in the center but the damping rate is small enough that the perturbative NOVA-K model can be applied. It is also clear from these quite different q profiles that the modes and damping rates found by NOVA-K are sensitive to the details of the q profile.

\subsection{Lower single null diverted $n=-4$ stable TAE with ramping toroidal field}

Stable TAE resonances were also excited in lower single null diverted plasmas with ramping toroidal field as shown in Figure 5. Three resonances are observed as the toroidal field ramps down and the density ramps up and down so that the center of the gap TAE frequency for q=1.5 passes through the constant antenna frequency three times. The plasma current was nearly 
constant at $\mathrm{I}_{\mathrm{p}}=0.77 \mathrm{MA}$ and $\mathrm{q}_{95}$ ramped from 5.8 down to 5.4 during this time window. The third resonance was found to have $n=-4$ in the ion direction with a frequency of $\mathrm{f}_{\mathrm{TAE}}=436 \mathrm{kHz}$ and a measured damping rate of $\gamma / \omega \approx-0.8 \%$.

This case was modeled with NOVA-K using q profiles calculated from EFIT and TRANSP (Figure 6), which are somewhat different due to the different boundary conditions in the two codes and the assumed parameterizations of the current density profile. The core plasma ion toroidal rotation was measured to be $\mathrm{f}_{\text {tor }} \approx 4 \mathrm{kHz}$ in the electron direction so that the mode frequency in the plasma frame should be $\mathrm{f}_{\text {plasma }} \approx \mathrm{f}_{\text {meas }}+16 \mathrm{kHz} \approx 452 \mathrm{kHz}$ for an $n=-4$ mode rotating in the ion direction. Modes were found for both q profiles in this frequency range (Table 2). For the TRANSP q profile, modes were found that bracket the measured frequency at 420.6 $\mathrm{kHz}$ with a total damping rate of $-1.06 \%$ and at $476.4 \mathrm{kHz}$ with a total damping rate of $-1.55 \%$, not very different from the measured damping rate of $-0.8 \%$. Three modes were found with the EFIT q profile with frequencies from $419.9 \mathrm{kHz}$ to $456.1 \mathrm{kHz}$. This frequency is close to the measured frequency with the calculated Doppler shift and had a total damping rate of $-0.7 \%$, which is almost identical to the measured damping rate. The main contributions to the damping rate were from continuum damping at the edge and from Landau damping on the background deuterium ions for both q profiles. So, for both of these q profiles, modes in the measured frequency range were found with damping rates close to the measured value.

The calculated radial structures and $n=4$ TAE gap structures for the mode that is closest to the experiment for each of the two q profiles are shown in Figure 7. Both modes interact with the continuum inside of $\sqrt{\Psi_{p o l}}$ between 0.4 and 0.45 . In the case of the EFIT q profile, there is a somewhat larger edge region of interaction with the continuum outside of $\sqrt{\Psi_{\text {pol }}} \sim 0.80$, while 
the edge interaction for the TRANSP q profile occurs slightly further out near $\sqrt{\Psi_{\text {pol }}} \sim 0.85$.

While the mode with the EFIT q profile agrees better with the experimental frequency and damping rate, there is stronger continuum interaction near the edge.

One of the goals here is to study the sensitivity of the NOVA-K continuum damping model to different plasma conditions. In this example, it is clear that both modes found in this section are beyond the limit of the applicability of the perturbation technique used for continuum damping calculations. It turns out that modes calculated for this case strongly interact with the continuum at the edge and propagate through several resonances with the continuum. Small changes in the density profile can result in significant variation in the calculated continuum damping due to an induced change in the continuum. Numerically, this happens if the resonance is near the tip of the local continuum, at the grid point of the radial mesh, or if the poloidal harmonic coupling is strong, which is typical for low to moderate n numbers. Analysis shows that strong interaction with the continuum in this case is due to the low $n$ number and the closed TAE gap. Thus, NOVA-K can not reliably compute the damping rate in this case. From this comparison, we can conclude that higher $\mathrm{n}$ modes in general are better suited for the modeling because of stronger localization inside the gap. On the other hand localized TAEs are less coupled to the plasma edge and thus to the driving antenna. It is surprising that the frequency match helps to choose the mode, which has significant presence at the edge and has a damping rate close to the measured one. Note, that the finite edge amplitude of the eigenmode is also possible if it propagates through the continuum as will be discussed in the next section. The application of NOVA-K also shows that the perturbative damping model needs to be extended for multiple resonances with the continuum by including FLR and other non-ideal effects to 
resolve the singularities near the resonances. It is possible that the actual q profile is different from those reconstructed by TRANSP and EFIT, which would result in a different gap structure or FLR effects adjust the TAE mode structure accordingly.

\subsection{Near double null inner wall limited $n=9$ stable TAE with sweeping active frequency}

By sweeping the active antenna frequency up and down through the center of the TAE gap frequency, multiple resonances can be excited in a single discharge. Figure 8a shows the calculated plasma equilibrium cross-section for a near double null inner wall limited plasma at the time when a TAE resonance is observed during active frequency sweeping. The frequency sweeping is shown in the spectrogram of a pick-up coil signal in Figure $8 \mathrm{~b}$ together with the time averaged poloidal field fluctuation amplitude and the calculated center of the gap TAE frequency for $\mathrm{q}=1.5$. The active frequency tracks the TAE frequency for a given q value through a digital control system that calculates the TAE frequency in real-time with analog measurements of the core line integrated density and toroidal field, assuming constant chord length, major radius, and impurity fraction. This gives an approximate value for the TAE frequency at $\mathrm{q}=1.5$ and although there is certainly error in the approximation compared to the actual value, it does get close enough for the sweep to cross the desired resonant frequency. A clear resonance at about $0.85 \mathrm{~s}$ was found to have a frequency of $f_{\text {meas }}=487 \mathrm{kHz}$. Fitting the phases of the residues of three closely toroidally spaced pick-up coil signals gives a clear linear fit to $n=9$ (Figure 9). The measured damping rate was found to be $\gamma / \omega \approx-1.5 \%$. Once again NOVA-K was run using the calculated q profiles from TRANSP and EFIT, but in addition, the sensitivity to the q profile was checked by artificially scaling the entire EFIT q profile by $0.9,1.0$, and 1.1 . While this does not preserve the correct edge q value, it does provide a simple means to check the sensitivity of the 
calculated mode structure and damping rate to relatively small changes in the overall q profile without changing the shape of the profile or other inputs to the code. Figure 10 shows the EFIT and TRANSP q profiles used in the calculations. Note that the TRANSP central q value is 0.54 , which is much lower than the EFIT $\mathrm{q}_{0}$ value, which is constrained to be near 1 . The measured core ion plasma toroidal rotation was $\sim 5 \mathrm{kHz}$ in the electron direction, so the mode frequency in the plasma frame should be approximately $\mathrm{f}_{\text {plasma }}=\mathrm{f}_{\text {meas }}-45 \mathrm{kHz} \approx 442 \mathrm{kHz}$. Note that given the relatively large toroidal mode number, a small change in the plasma rotation will make a much larger change in the Doppler shifted frequency, so there is a larger uncertainty in the mode frequency in the plasma frame. Table 3 shows the calculated damping rates for the TRANSP $q$ profile and for the three scaled EFIT q profiles. Eigenmodes with calculated frequencies in the range from $420 \mathrm{kHz}$ to $450 \mathrm{kHz}$ were found for each of these q profiles, but the calculated damping rate was found to be quite sensitive to q, varying by nearly an order of magnitude with these changes in $\mathrm{q}$. The variation in the damping rates is again from the different radial localization of these modes with the respect to the TAE gap.

For the TRANSP q profile, the low central q value leads to a less radially aligned gap structure and thus to modes with fairly strong continuum interaction in both the inner half of the plasma and out near the plasma edge. Figure 11a shows the radial profile of the eigenfunction for a mode at $449.5 \mathrm{kHz}$. There is strong continuum interaction inside $\sqrt{\Psi_{p o l}} \leq 0.6$ and outside $\sqrt{\Psi_{p o l}} \geq 0.9$ as can be seen in Figure $11 \mathrm{~b}$ showing the $n=9$ TAE gap structure. This mode had a calculated damping rate of $\gamma / \omega=-1.48 \%$, in agreement with the measured value. 
The NOVA-K calculations found a more open TAE gap structure for $0.9,1.0$, and 1.1 times the EFIT q profile. For the EFIT q profile multiplied by 0.9 , a mode was found at 440.0 $\mathrm{kHz}$ with a damping rate of $-1.2 \%$, very close to the measured damping rate. For the EFIT $\mathrm{q}$ profile, the mode found with the frequency closest to the Doppler shifted measured mode frequency had a frequency of $446.3 \mathrm{kHz}$. This mode had a calculated damping rate of $-0.33 \%$, which is considerably lower than the measured value of $-1.5 \%$. However, when the EFIT q profile was multiplied by 1.1 , the closest mode to match the experiment was found at $435.8 \mathrm{kHz}$ which had a damping rate of only $-0.17 \%$, nearly an order of magnitude smaller than the measured damping rate. The calculated radial profiles of the eigenfunctions and the TAE gap structures for these three scaled EFIT q profiles are shown in Figure 12. For 0.9 times the EFIT q profile, the mode amplitude near the edge is particularly large where there is strong interaction with the continuum that leads to a larger total damping rate. For the EFIT q profile, the edge interaction is not as large, but there is interaction with the continuum for $\sqrt{\Psi_{\text {pol }}}<0.5$, which leads to an intermediate damping rate. At 1.1 times the EFIT q profile, there is some continuum interaction at mid radius as well as near the edge, but the resulting damping rate is much smaller. This clearly shows how the continuum damping, in particular, is quite sensitive to details of the $\mathrm{q}$ profile. The case with the scaled EFIT q profiles is interesting as it shows that the TAE modes can propagate through and escape strong interaction with the continuum under certain conditions as discussed in Ref.[25]. It can happen if the continuum band is very narrow in the radial direction or when there is only one resonance with the continuum, so that the envelope of the high-n TAE mode structure can have a node in its structure at the point of its intersection with the continuum. This is the case for the TAEs corresponding to the EFIT q-profile and 1.1 times 
the EFIT q-profile, where the edge mode structure falls within the EAE gap so that the mode structure is part TAE and part EAE, or a double gap mode [25]

The reason for the eigenmode propagation outside the TAE gap can be understood as follows. Scaled EFIT q-profiles result in a similar TAE gap envelope, particularly near the edge, except that the radial positions of the $\mathrm{m}, \mathrm{m}+1$ couplets are shifted and the center of the TAE gap is moved according to $\omega_{\mathrm{TAE}} \sim 1 / \mathrm{q}$. Thus, if the external antenna frequency (or expected eigenmode frequency) is fixed, scaling the q profile is almost equivalent to changing the frequency for a fixed q-profile, particularly near the edge. Thus, going from a q-profile scaling factor of 0.9 to 1 to 1.1 moves the antenna frequency up with regard to the TAE gap envelope at each radial location. At 0.9 times the EFIT q-profile, as can be seen from the continuum Figure 12 (a), the radial distance between the TAE/EAE continuum and the edge is smallest (from $\mathrm{r} / \mathrm{a}=0.95$ to 1 ), so that the eigenmode can not be formed inside the EAE gap. That is, the half wavelength of the mode radial envelope does not fit within that part of the EAE gap. For both 1 and 1.1 times the EFIT q-profile, on the other hand, half of the eigenmode envelope wavelength fits nicely into the EAE gap. As a result the eigenmode escapes strong damping and propagates through the continuum, which allows it to be coupled to the antenna and be measured by the Mirnov coils at the wall. 


\section{Conclusions}

Detailed analyses with the NOVA-K code of several actively excited stable TAEs in Alcator CMod have shown that reasonable agreement between the measured and calculated total damping rates can be obtained for moderate toroidal mode numbers with $4 \leq n \leq 9$, for q profiles obtained with the EFIT or TRANSP codes. Since the q profile is not measured in C-Mod, a sensitivity analysis of the calculated damping rate to small changes in the q profile was performed and it was found as expected to be very sensitive to changes in $\mathrm{q}$. The calculated damping rate can increase or decrease by as much as an order of magnitude with only $10 \%$ changes in q. This sensitivity appears to be due to changes in how well radially aligned the TAE gap is and thus in the radial location of the interaction of the mode with the continuum such that the mode may be less affected by continuum damping at mid radii or be more strongly damped near the edge, depending on changes in the q profile. The Motional Stark Effect diagnostic on C-Mod with the diagnostic neutral beam is unable to measure the q profile to an accuracy of $10 \%$. A more accurate measurement of the q profile will be necessary for more precise validation of the NOVA-K continuum damping rate model with measured stable TAE damping rates. It is possible that in cases when the gap is closed, TAEs have relatively small damping. It means that to analyze them, NOVA-K should be extended to include FLR effects to resolve the TAE mode structure in the vicinity of the resonance with the continuum. In general, the NOVA-K continuum damping model seems to be working reasonably well for moderate- $n$ modes, for which the continuum damping rate is expected to be smaller due to their relative localization. It follows from the analysis above that the frequency match is not the only criterion for the mode to be chosen out of the linear simulations. A second criterion is that the eigenmode must have finite amplitude at the edge of the plasma. This becomes increasingly important for higher 
toroidal mode numbers when the number of eigenmodes in the simulations increases in

proportion to $\mathrm{n}$.

\section{Acknowledgements}

We would like to thank Prof. Ambrogio Fasoli for invaluable discussions related to this work and William Burke for all of his efforts to get the amplifiers working well to excite these modes. We would also like to thank the C-Mod team for keeping the tokamak running well. This work is supported by the US Department of Energy contract DE-FC02-99ER54512.

\section{References}

[1] Wong K. L., Fonck R. J., Paul S. F., et al, 1991 Phys. Rev. Lett. 66, 1874.

[2] Heidbrink W. W., Strait E. J., Doyle E., and Snider R., 1991 Nucl. Fusion 31, 1635.

[3] Saigusa M., Kimura H., Moriyama S., Neyatani Y., Fujii T., et al, 1995 Plasma Phys. Cont. Fus. 37, 295.

[4] Fasoli A., Lister J.B., Sharapov S.E., et al, 1995 Nucl. Fusion 35, 1485.

[5] Weller A., Spong D. A., Jaenicke R., Lazaros A., Penningsfield F. P., Sattler S., W7-AS Team, and NBI Group, 1994 Phys. Rev. Lett. 72, 1220.

[6] Candy J. and Rosenbluth M. N., 1995 Nucl. Fusion 35, 1069.

[7] Gorelenkov N. N., Bernabei S., Cheng C. Z., et al, 2000 Nucl. Fusion 40, 1311.

[8] Betti R. and Freidberg J. P., 1992 Phys. Fluids B 4, 1465.

[9] Fulvio Zonca and Liu Chen, 1993 Phys. Fluids B 5, 3668.

[10] Fu G. Y., Cheng C. Z., Budny R., Chang Z., Darrow D. S., et al, 1996 Phys. Plasmas 3, 4036.

[11] Rosenbluth M. N. and Rutherford P. H., 1975 Phys. Rev. Lett. 34, 1428.

[12] Cheng C. Z., Chen L., and Chance M. S., 1985 Ann. Phys (N.Y.) 161, 21.

[13] Fu G. Y., and Van Dam J. W., 1989 Phys. Fluids B 1, 1949.

[14] Fasoli A., Borba D., Bosia G., Campbell D. J., et al, 1995 Phys. Rev. Lett. 75, 645.

[15] Testa D., Fasoli A., 2001 Nucl. Fus. 41, 809.

[16] Fasoli A., Testa D., Sharapov S., Berk H. L., Breizman B., et al, 2002 Plasma Phys. Cont. Fus. 44, B159.

[17] Testa D., Fu G. Y., Jaun A., Fasoli A., Sauter O., et al, 2003 Nucl. Fusion 43, 479.

[18] Fu G.Y., Berk H.L., and Pletzer A., Phys. Plasmas 12 (2005) 082505

[19] Lauber Ph., Günter S. and Pinches S.D., Phys. Plasmas 12 (2005) 122501

[20] Snipes J. A., Schmittdiel D., Fasoli A., Granetz R. S., and Parker R. R., 2004 Plasma Phys. Cont. Fus. 46, 611.

[21] Snipes J. A., Schmittdiel D. A., Fasoli A., Burke W., Granetz R. S., et al, 2003 Proc. 30th EPS Conf on Contr. Fusion and Plasma Phys., St. Petersburg, 7-11 July 2003 ECA 27A, P4.93.

[22] Snipes J. A., Basse N., Boswell C., Edlund E., Fasoli A., et al, 2005 Phys. Plasmas 12, 056102. 
[23] Cheng C.Z., 1992 Phys. Reports 2111.

[24] Fu G. Y.,Cheng C. Z., K.-L.Wong, 1993 Phys. Fluids B 5, 4040.

[25] Gorelenkov N.N., 2005 Phys.Rev.Lett. 95265003.

[26] Burke W., Cochran W., Schmittdiel D., Snipes J., Zhong X., 2003 Proc. $20^{\text {th }}$ IEEE/NPSS Symposium on Fusion Engineering, 363.

[27] Porkolab Miklos, Rost J Chris, Basse Nils, Dorris James, Edlund Eric, et al, 2006 IEEE Transactions on Plasma Science, 0093-3813 to be published.

[28] Rice J. E., Marmar E. S., 1990 Rev. Sci. Instrum., 61, 2753.

[29] Berk, H.L., Van Dam, J.W., Guo, Z., Lindberg, D.M., Phys.Fluids B v.4 (1992) 1806.

[30] Lao L. L., St John H., Stambaugh R. D., Kellman A. G., and Pfeiffer W., 1985 Nucl Fus 25, 1611

[31] Hirshman S.P., Schwena U., Nuhrenberg J., 1990 J. Comp. Phys. 87, 396.

\section{Figure Captions}

Fig. 1. (a) Magnetic equilibrium flux contours of the plasma cross-section showing the locations of the active MHD antennas. (b) Photo of the active MHD antennas inside C-Mod. (c) Geometric calculation of the toroidal mode spectrum of an active MHD antenna showing that a broad spectrum of modes are excited.

Fig. 2. (a) Magnetic equilibrium flux contours showing an inner wall limited plasma crosssection at the time of a TAE resonance. (b) Fourier spectrogram of a magnetic pick-up coil signal together with time averaged amplitude of the poloidal field fluctuations and the calculated center of the TAE gap frequency (solid line) compared with the active antenna frequency (dashed line) showing that a resonant peak occurs just after the calculated TAE frequency passes through the antenna frequency.

Fig. 3. EFIT calculated q profile compared with a flat q profile out to $\sqrt{\Psi_{p o l}} \sim 0.7$ used in NOVA-K to calculate the TAE damping rate for the resonance shown in Fig. 2.

Fig. 4. (a) Alfvén eigenmode dispersion relation for the $n=-4$ mode as a function of the radial coordinate $\sqrt{\Psi_{\text {pol }}}$ showing that the $427.4 \mathrm{kHz}$ mode lies in the TAE gap. (b) Radial eigenmode structure calculated with NOVA-K of the $\mathrm{n}=-4$ mode at $427.4 \mathrm{kHz}$ found using the equilibrium for the resonance measured in Fig. 3.

Fig. 5. (a) Magnetic equilibrium flux contours for a lower single null plasma at the time of a stable TAE resonance. (b) Fourier spectrogram of a poloidal field pick-up coil signal showing three clear TAE resonances together with time averaged poloidal field perturbation, the changing line averaged density and toroidal field, which result in a changing center of the TAE gap frequency for $\mathrm{q}=1.5$ compared to the constant active antenna frequency.

Fig. 6. EFIT and TRANSP calculated q profiles at the time of the third resonance in Fig. 5. 
Fig. 7. (a) NOVA-K calculated TAE gap structure for $n=4$ with a TRANSP calculated q profile. (b) NOVA-K calculated eigenfunction radial profile for an $n=4$ mode at $476.4 \mathrm{kHz}$ with a TRANSP q profile. (c) NOVA-K calculated TAE gap structure for $n=4$ with an EFIT calculated q profile. (d) NOVA-K calculated eigenfunction radial profile for an $n=4$ mode a $456.1 \mathrm{kHz}$ with an EFIT q profile.

Fig. 8. (a) Magnetic equilibrium flux contours for a near double null inner wall limited plasma at the time of a stable TAE resonance. (b) Fourier spectrogram of a poloidal field pick-up coil signal together with the time average of the poloidal field fluctuations and the center of the TAE gap frequency for $\mathrm{q}=1.5$ compared with the sweeping active antenna frequency showing a resonance as the sweeping frequency passes through the TAE frequency.

Fig. 9. Linear fit to the phases of the residues of the transfer functions of three toroidally displaced poloidal field pick-up coil signals indicating a toroidal mode number of $n=-9$.

Fig. 10. TRANSP and EFIT calculated q profiles as a function of the square root of the normalized poloidal flux radial coordinate for the shot and time of the resonance in Fig. 8.

Fig. 11. (a) NOVA-K calculated TAE gap structure for $n=9$ with a TRANSP calculated q profile. (b) NOVA-K calculated eigenfunction radial profile for an $n=9$ mode at $449.5 \mathrm{kHz}$ with a TRANSP calculated q profile.

Fig. 12. NOVA-K calculated TAE gap structure and radial eigenfunctions for the closest $n=9$ mode to the experimentally measured frequency (a) and (b) with the EFIT calculated q profile multiplied by 0.9 , (c) and (d) with the EFIT q profile, and (e) and (f) with the EFIT q profile multiplied by 1.1 .

\section{Table Captions}

Table 1. NOVA-K calculated damping rates for the measured stable TAE resonance from Figure 2 using the two q profiles shown in Figure 3.

Table 2. NOVA-K calculated damping rates for the third stable TAE resonance in Figure 5 using the two q profiles shown in Figure 6.

Table 3. NOVA-K calculated damping rates for the measured stable TAE resonance centered about $0.85 \mathrm{~s}$ in Figure 8 using the TRANSP q profile and the EFIT q profile scaled by a factor of $0.9,1$, and 1.1 . 
EFIT q profile, $q 0=0.905,1021003013, t=1.0-1.1 \mathrm{~s}, \mathrm{n}=4, \mathrm{f}=440 \mathrm{kHz}, \gamma / \omega=-3.0 \%$ Freq $(\mathrm{kHz})$ e-collisions thermal e D C continuum Total $\gamma / \omega(\%)$

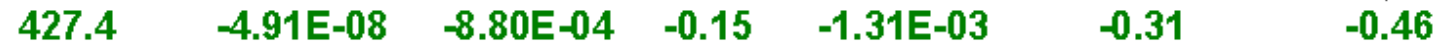

FLA T q profile, q0 $=0.95$

Freq $(\mathrm{kHz})$ e-collisions thermal e D C continuum Total $\gamma / \omega(\%)$

$\begin{array}{lllllll}457.2 & -1.15 E-08 & -5.74 E-04 & -0.11 & -3.39 E-04 & -0.08 & -0.19\end{array}$

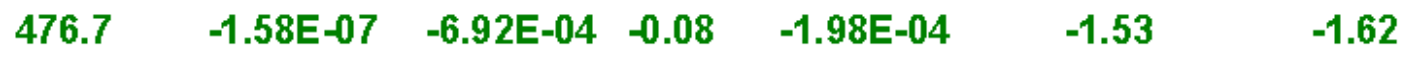

Table 1. 
TRANSP q profile, $q 0=0.82,1030520026$, $t=0.8-0.88 \mathrm{~s}, \mathrm{n}=4, \mathrm{f}=452 \mathrm{kHz}, \gamma / \omega=-0.8 \%$ Freq $(\mathrm{kHz})$ e-collisions thermal $\mathrm{e}$ $420.6 \quad-6.06 \mathrm{E}-07 \quad-1.76 \mathrm{E}-03$ 476.4 $-1.35 \mathrm{E}-07 \quad-1.31 \mathrm{E}-03$

D C continuum Total (\%)

$\begin{array}{llll}-0.21 & -7.53 E-04 & -0.84 & -1.06\end{array}$

EFIT q profile, $q 0=1.04$

Freq $(\mathrm{kHz})$ e-collisions thermal e D C continuum Total (\%) $\begin{array}{lllllll}419.9 & -3.747 \mathrm{E}-07 & -1.74 \mathrm{E}-03 & -0.23 & -8.24 \mathrm{E}-04 & -12.80 & -13.03\end{array}$ $\begin{array}{lllllll}431.6 & 3.806 \mathrm{E}-07 & -2.77 \mathrm{E}-03 & -0.28 & -5.78 \mathrm{E}-04 & -2.60 & -2.88\end{array}$ $\begin{array}{lllllll}456.1 & 3.40 \mathrm{E}-07 & -2.75 \mathrm{E}-03 & -0.26 & -3.22 \mathrm{E}-04 & -0.44 & -0.70\end{array}$

Table 2. 
TRANSP q profile, $q 0=0.54,1050615011, t=0.83-0.88 \mathrm{~s}, \mathrm{n}=-9, \gamma / \mathrm{o}=-1.5 \%, \mathrm{f}=442 \mathrm{kHz}$ Freq $(\mathrm{kHz})$ e-collisions thermale $\mathrm{D}$ C continuum Total $/ \mathrm{o}(\%)$ $\begin{array}{lllllll}449.5 & -9.45 E-07 & -1.14 \mathrm{E}-03 & -0.05 & -1.07 \mathrm{E}-03 & -1.43 & -1.48\end{array}$

EFIT q profile ${ }^{\star} 0.9, \mathrm{q} 0=0.9$

$\begin{array}{ccccccc}\text { Freq }(\mathrm{kHz}) & \text { e-collisions } & \text { thermal e } & \mathrm{D} & \mathrm{C} & \text { continuum } & \text { Total } \gamma / \boldsymbol{m}(\%) \\ 440.0 & -1.21 \mathrm{E}-06 & -2.18 \mathrm{E}-03 & -0.09 & -6.80 \mathrm{E}-04 & -1.11 & -1.20 \\ 445.6 & -4.46 \mathrm{E}-07 & -8.08 \mathrm{E}-04 & -0.05 & -1.24 \mathrm{E}-03 & -1.00 & -1.05\end{array}$

EFIT q profile q0 $=1.0$

Freq $(\mathrm{kHz})$ e-collisions thermal e $\mathrm{D} \quad \mathrm{C}$ continuum Total ris (\%) $\begin{array}{lllllll}422.5 & -2.91 \mathrm{E}-07 & 0.00 & -0.03 & -1.18 \mathrm{E}-03 & -0.65 & -0.68\end{array}$ $\begin{array}{lllllll}446.3 & -3.90 \mathrm{E}-07 & 0.00 & -0.04 & -1.08 \mathrm{E}-03 & -0.28 & -0.33\end{array}$

EFIT q profile *1.1, q0 $=1.1$

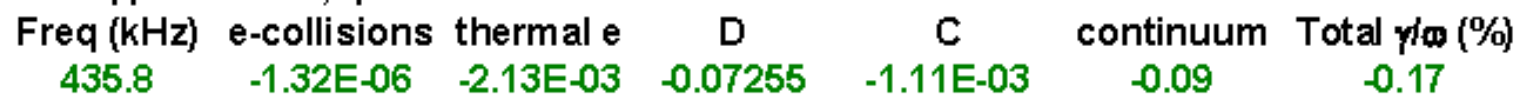

Table 3 . 


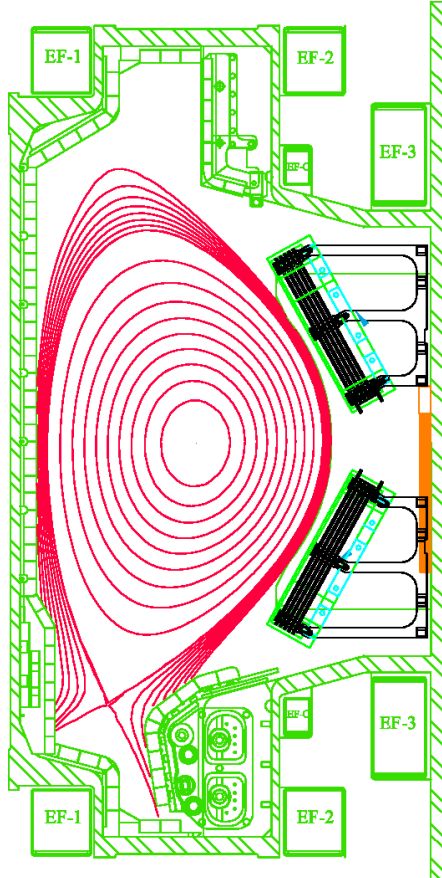

(a)

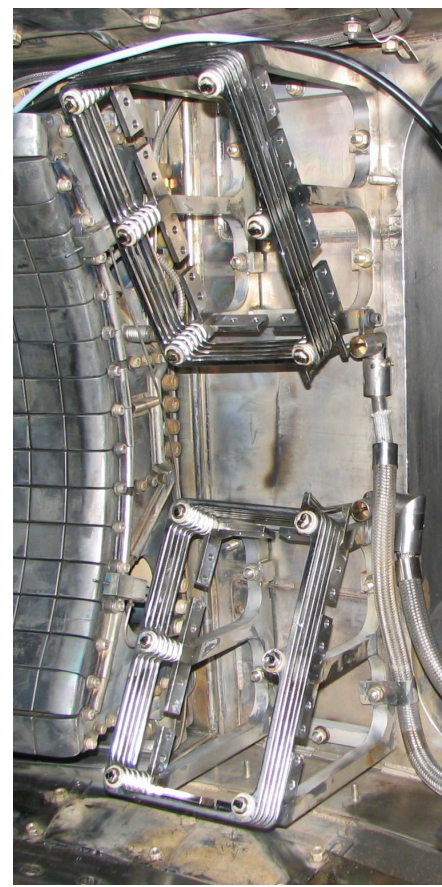

(b)
Active MHD Antenna Spectrum

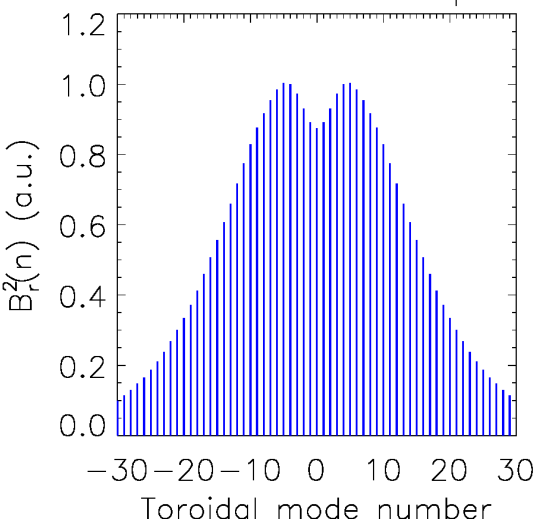

(c)

Fig. 1. 


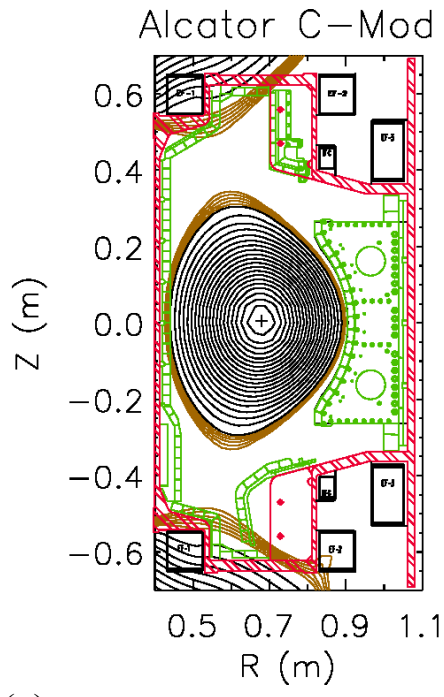

(a)

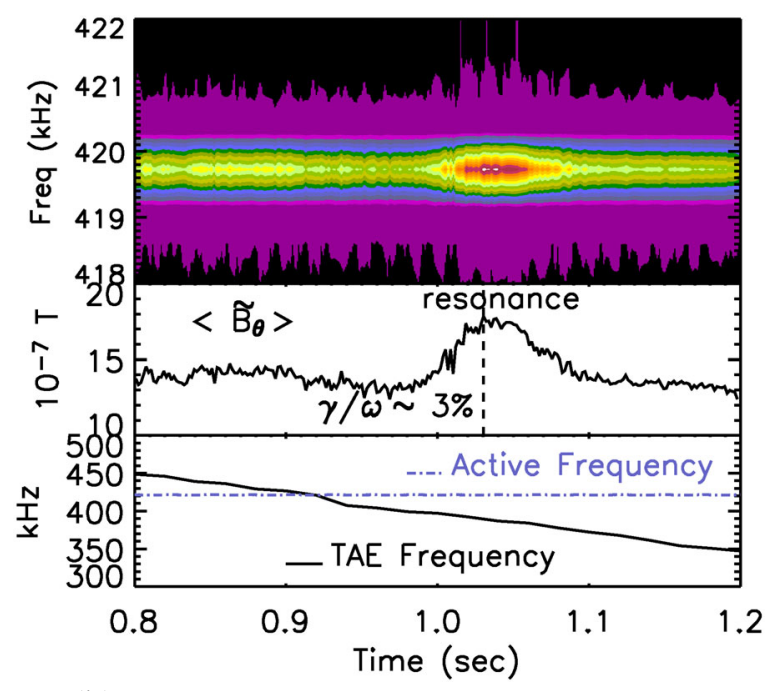

(b)

Fig. 2. 


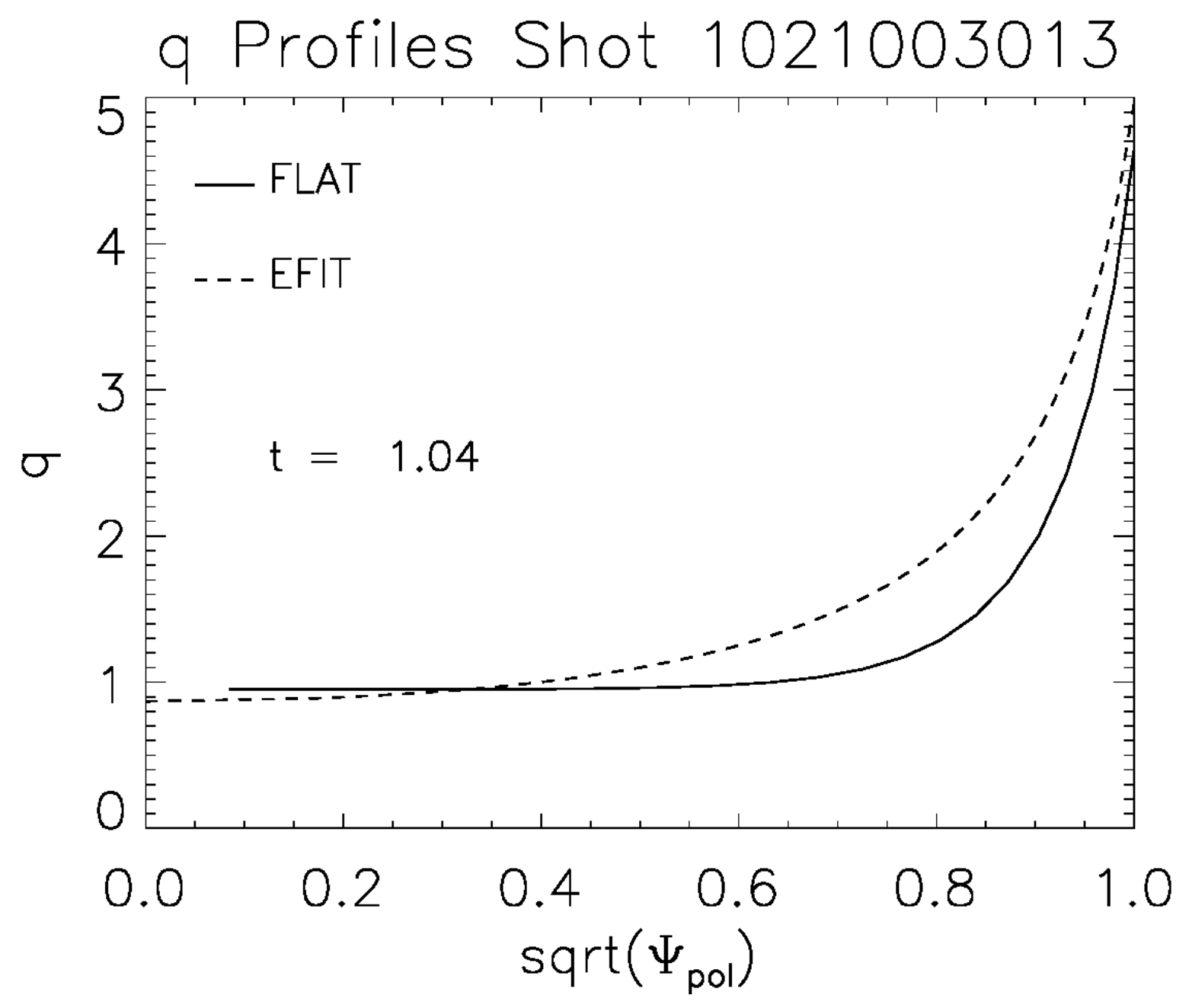

Fig. 3. 


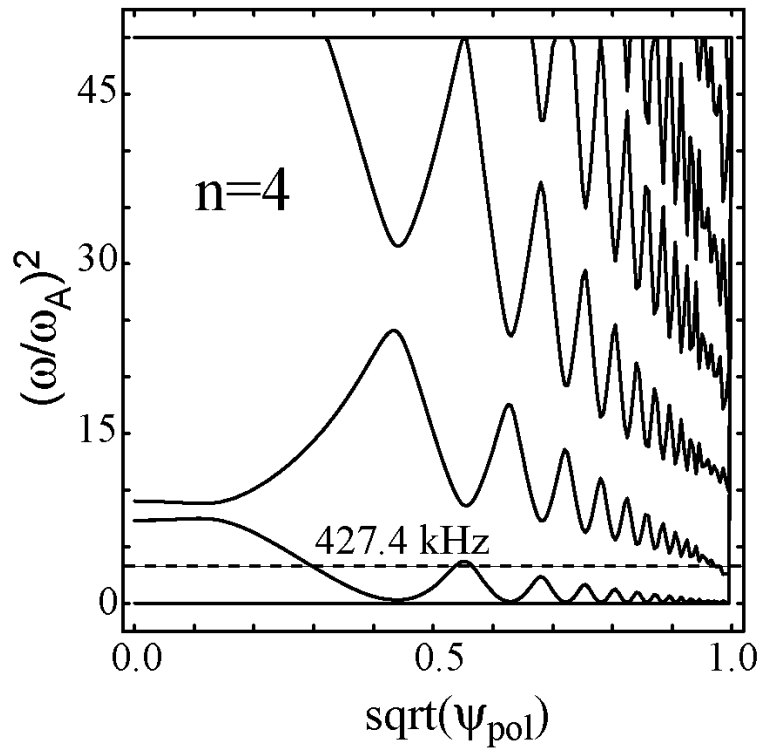

(a)

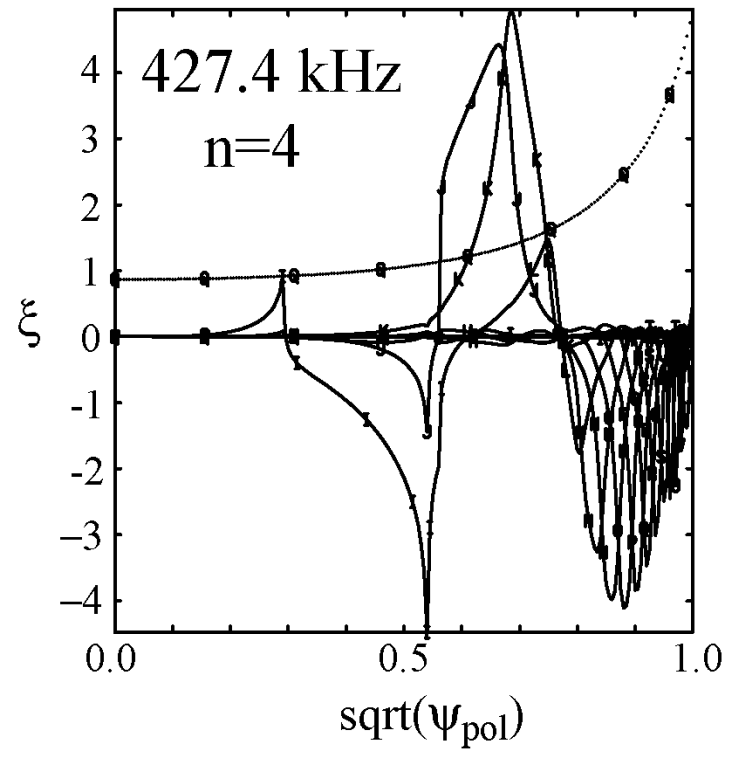

(b)

Fig. 4. 


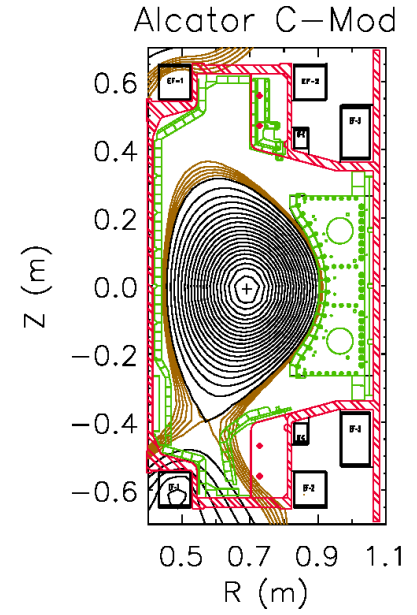

(a)

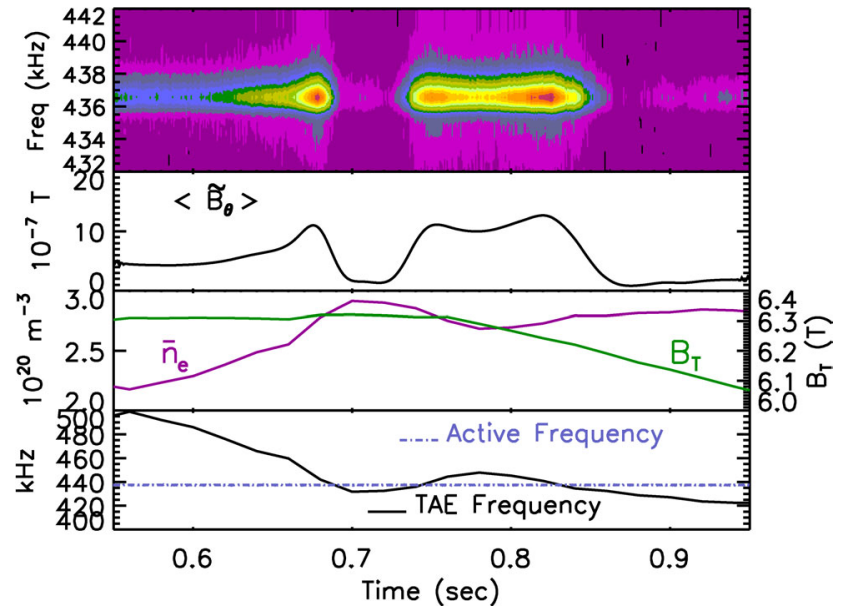

(b)

Fig. 5. 


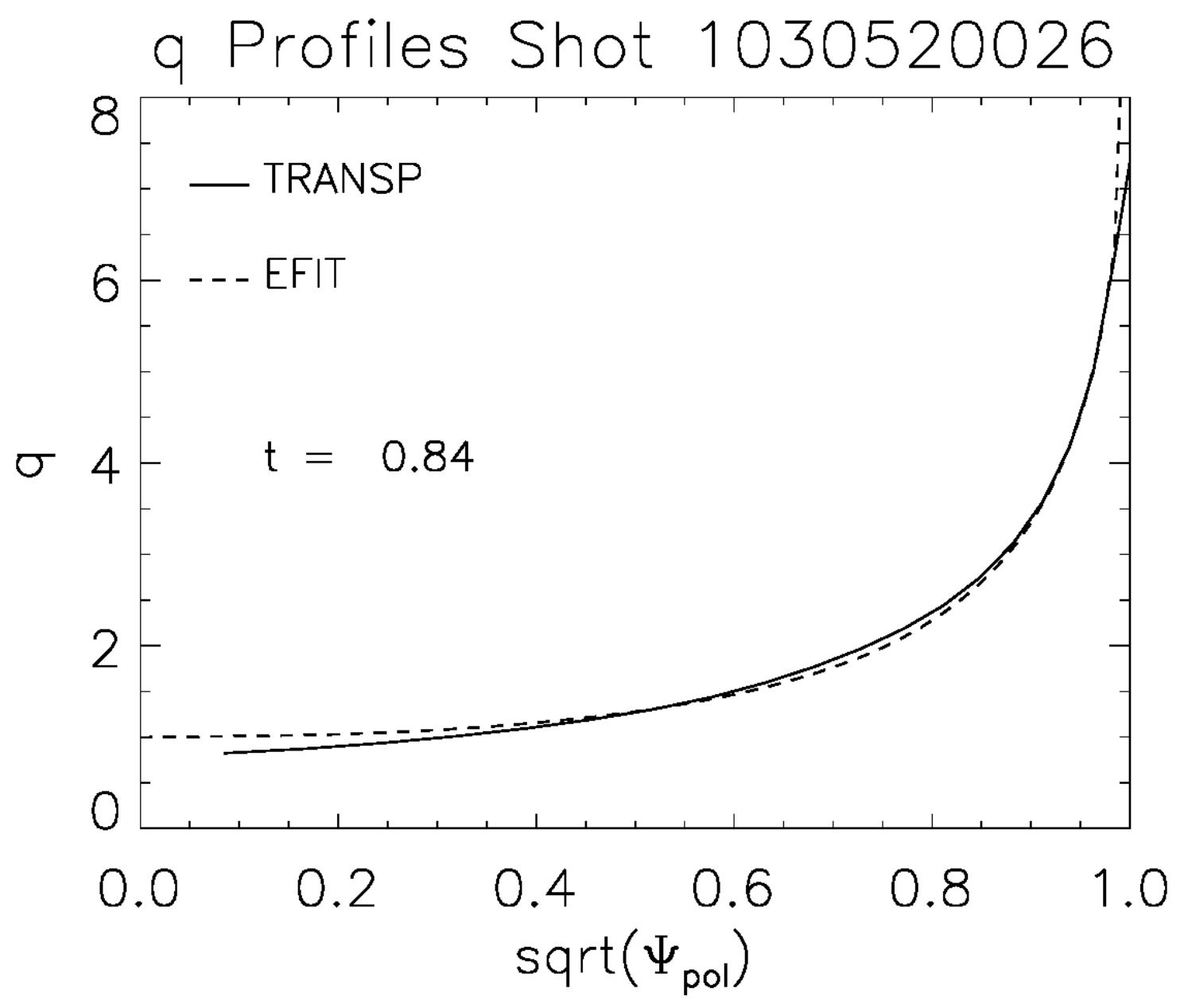

Fig. 6. 


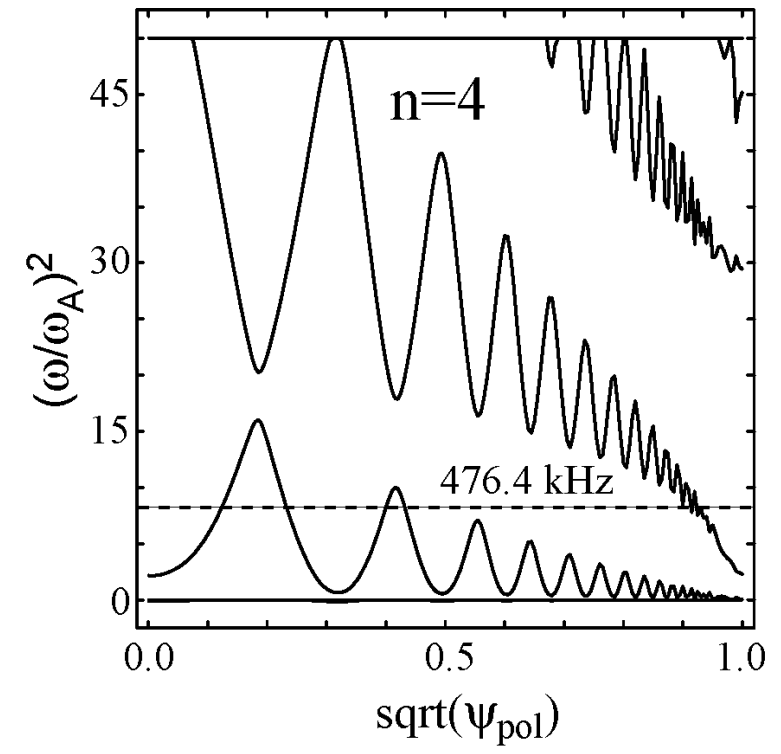

(a)

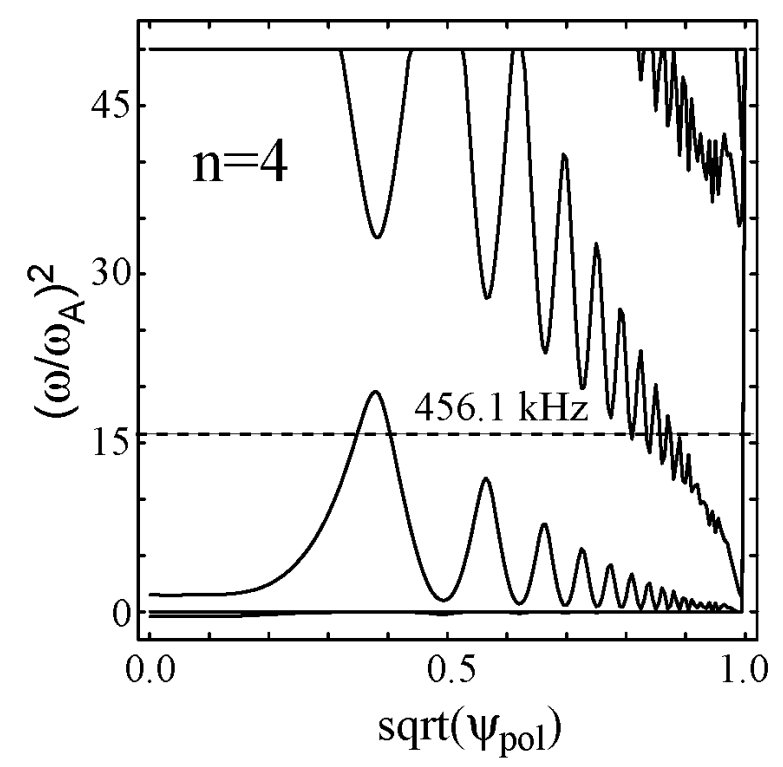

(c)

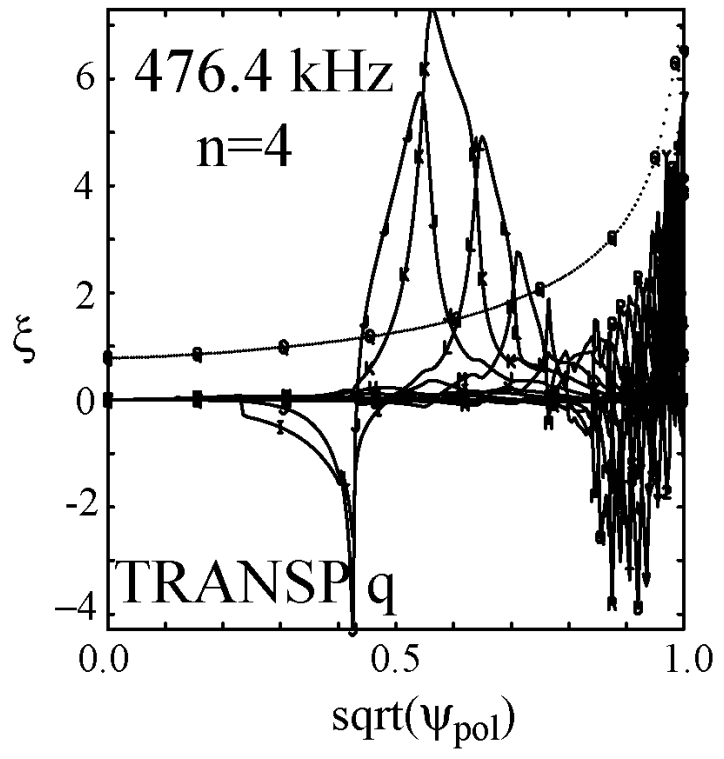

(b)

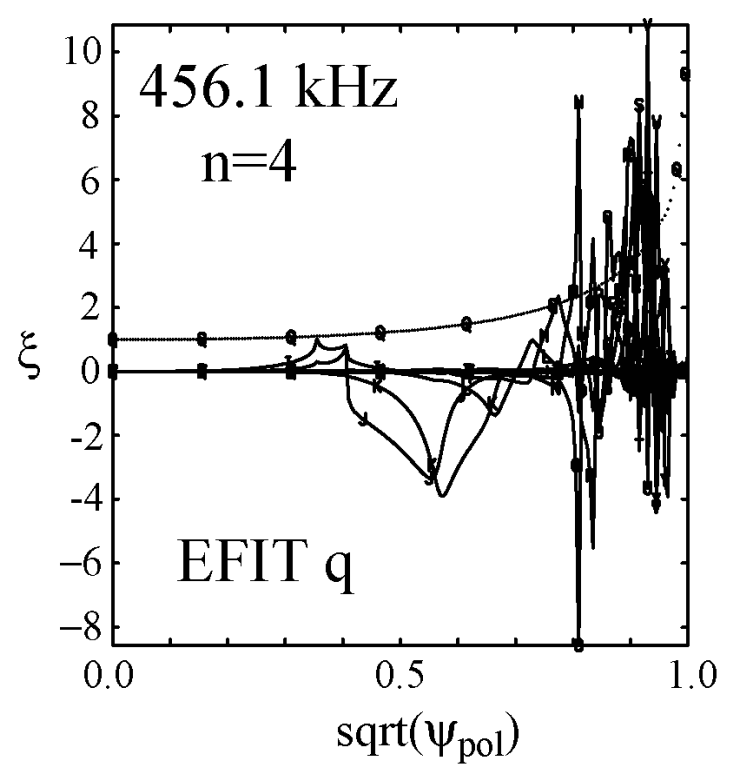

(d)

Fig. 7. 


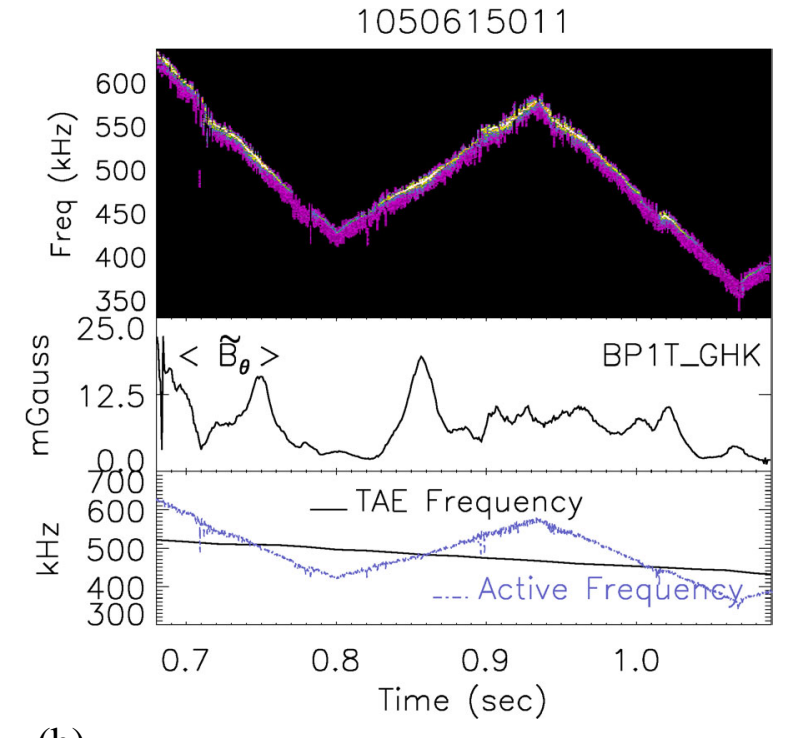

(b)

Fig. 8.

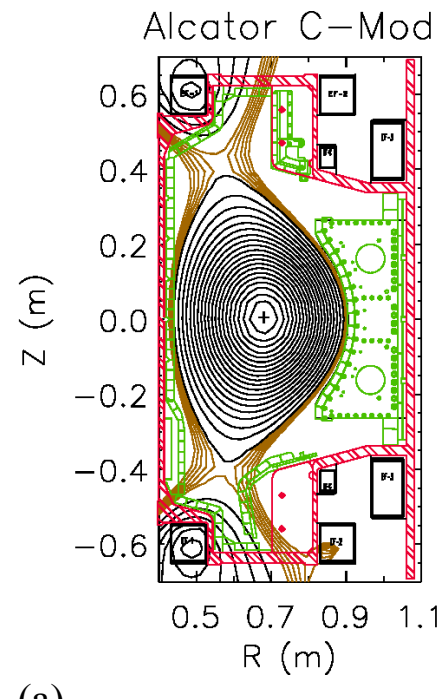

(a)
1050615011

$I_{p}(M A)=-0.827$

$B_{T}(T)=-5.64$

Time $(s)=0.840$

$R_{\text {axis }}(\mathrm{m})=0.667$

$Z_{\text {oxis }}(\mathrm{cm})=0.18$

$a(m)=0.227$

$\kappa=1.683$

$\delta_{u}=0.382$

$\delta_{1}=0.361$

$\mathrm{I}_{\mathrm{i}}=1.40$

$\mathrm{q}_{\psi}(95)=5.25$

$\operatorname{gap}_{\lim }(\mathrm{cm})=-0.09$

$\operatorname{gap}_{\text {in }}(\mathrm{cm})=0.00$

gapout $(\mathrm{cm})=1.16$ 


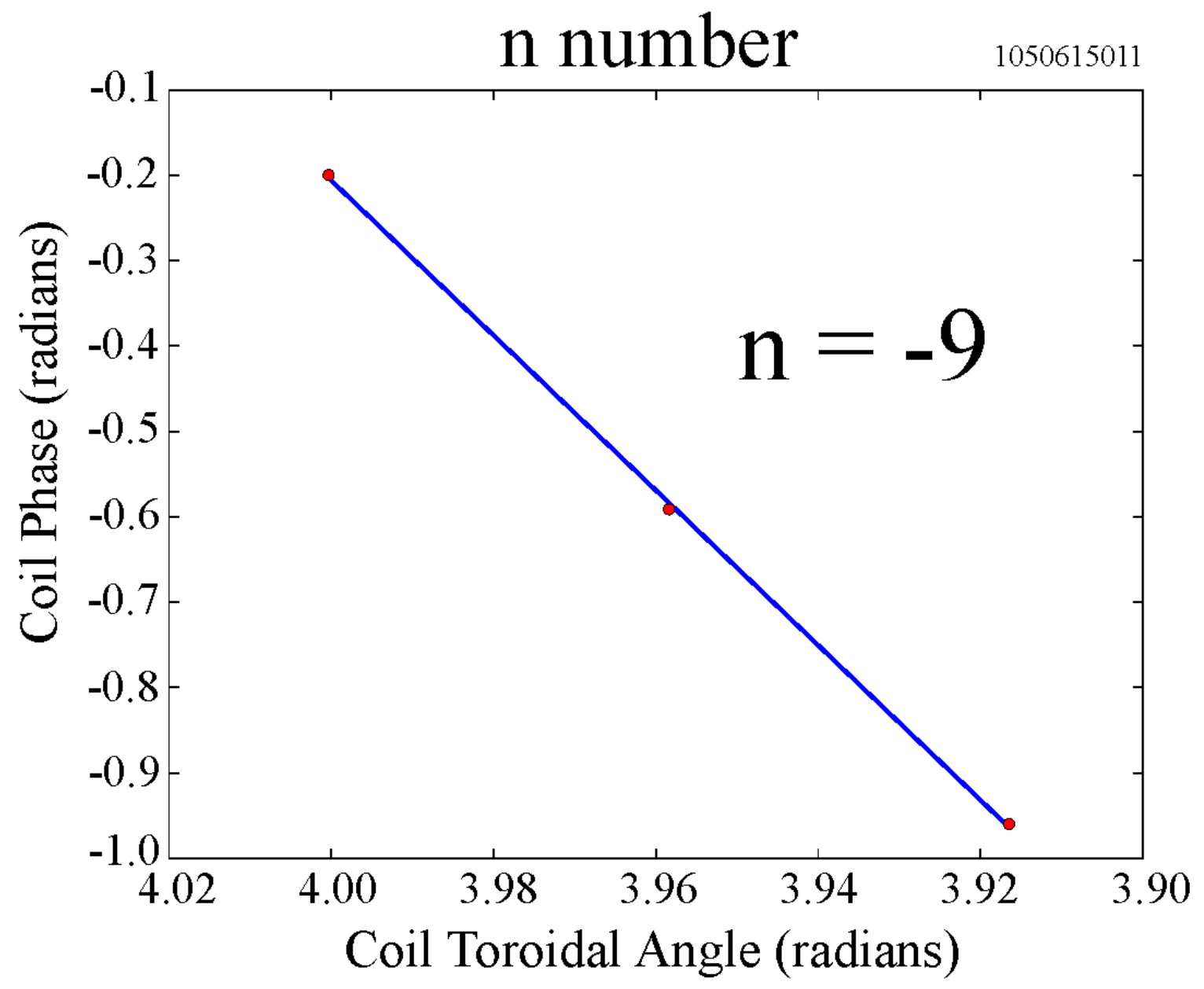

Fig. 9. 


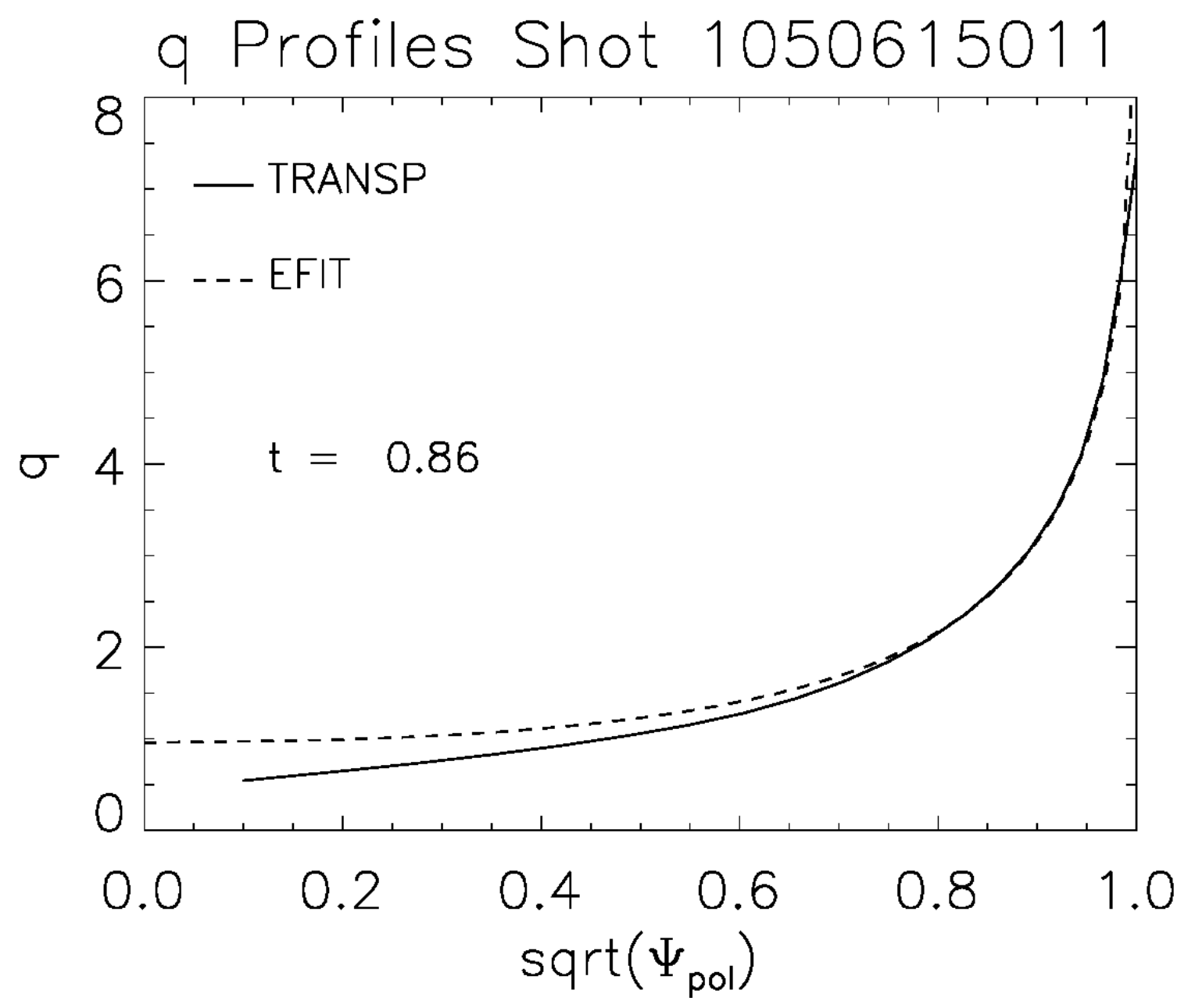

Fig. 10 . 


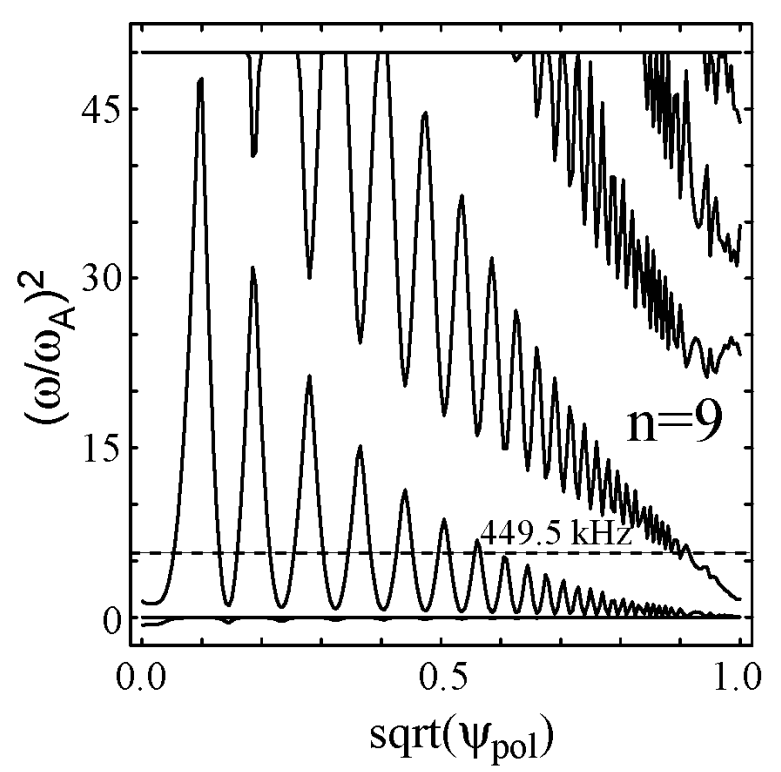

(a)

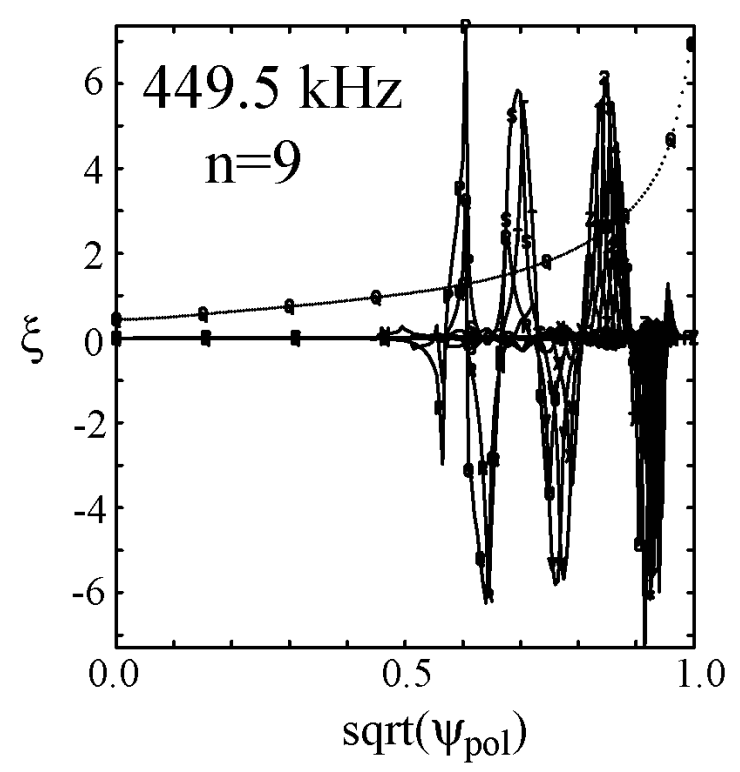

(b)

Fig. 11. 


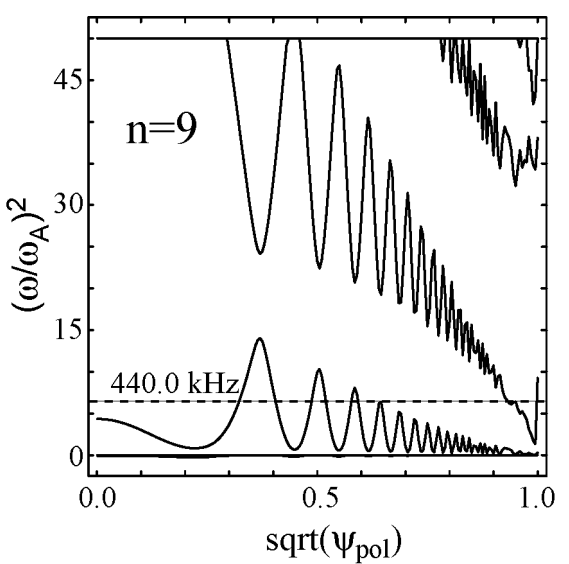

(a)

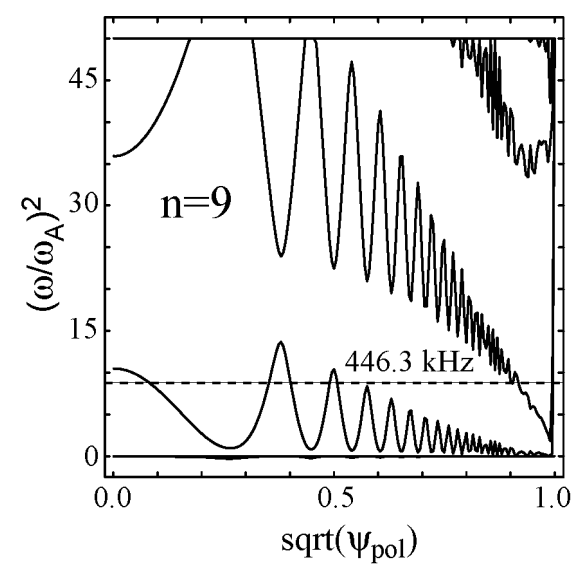

(c)

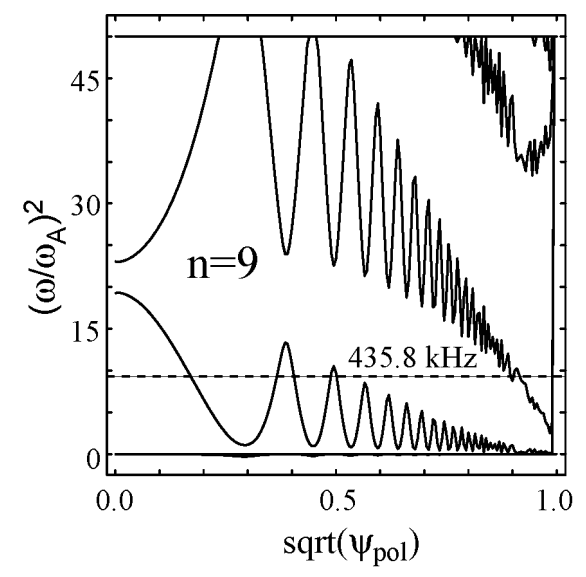

(e)

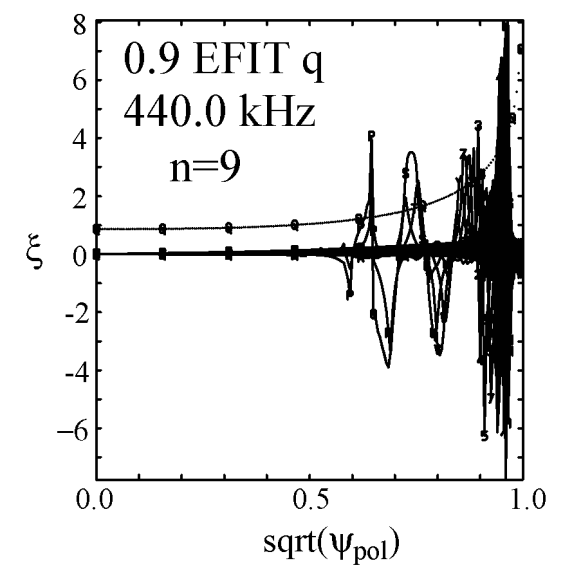

(b)

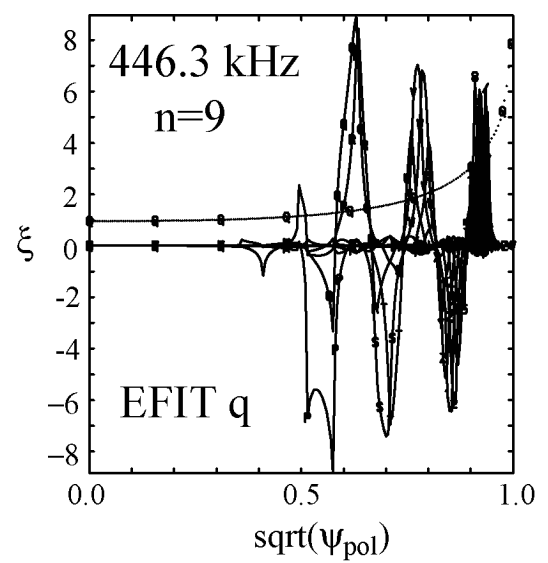

(d)

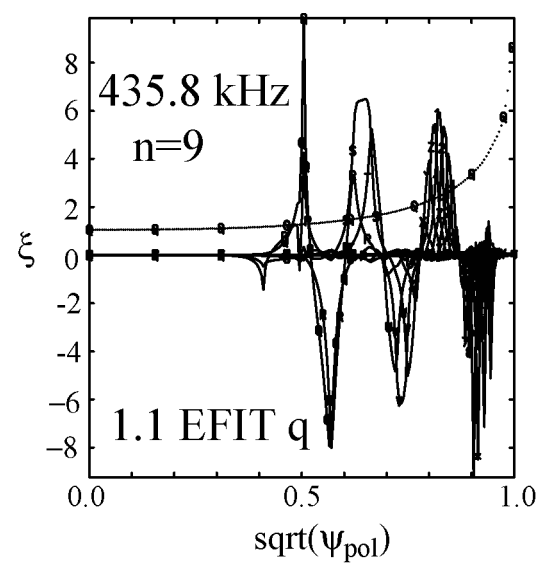

(f)

Fig. 12 . 